\title{
AIM2 contributes to the maintenance of intestinal integrity via Akt and protects against Salmonella mucosal infection
}

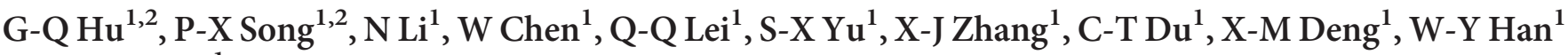 \\ and Y-J Yang
}

The mechanism regulating the gastrointestinal epithelial barrier remains poorly understood. We herein demonstrate that Absent in melanoma-2 (AIM2) contributes to the maintenance of intestinal barrier integrity and defense against bacterial infection. AIM2-deficient mice displayed an increased susceptibility to mucosal but not systemic infection by

Salmonella typhimurium, indicating a protective role for AIM2 in the gastrointestinal tract. In a Salmonella colitis model, compared with wild-type mice, AIM2 ${ }^{-1-}$ mice exhibited more severe body weight loss, intestinal damage, intestinal inflammation, and disruption of basal and activated epithelial cell turnover. In vivo and in vitro data showed that AIM2 restricted the early epithelial paracellular invasion of Salmonella and decreased epithelial permeability. The decreased epithelial barrier in AIM2 ${ }^{-1-}$ mice might be attributed to the altered expression of tight junction proteins that contribute to epithelial integrity. AIM2 promoted the expression of tight junction proteins through Akt activation. Together, these results suggest that AIM2 is required for maintaining the integrity of the epithelial barrier.

\section{INTRODUCTION}

The gastrointestinal (GI) tract is covered with a single-layered columnar epithelium that functions as a physical and biological barrier protecting the host against resident and foreign pathogens present in the gut. ${ }^{1}$ To gain access to the host, pathogens must first breach the intestinal epithelial barrier. ${ }^{2}$ Thus, understanding the mechanisms regulating this barrier is of fundamental importance to prevent microbial infection and disease progression.

The Salmonella enterica serovar typhimurium (Salmonella) is a prevalent foodborne pathogen that causes enteric disease in diverse animals and humans. After oral ingestion, Salmonella initially breaches the epithelial lining, triggers mucosal inflammation, disseminates into the mesenteric lymph nodes (MLNs), and even reaches and colonizes systemic sites such as the spleen and liver. Additionally, there are many non-typhoidal Salmonella strains typically cause self-limiting subacute/acute gastroenteritis in immune-competent individuals; the infection is in most cases confined to the
GI tract, and dissemination to the liver and spleen is atypical. Salmonella thus serves as a good model for studying the intestinal epithelial barrier against bacterial pathogens. ${ }^{3,4}$

Absent in melanoma-2 (AIM2), a member of the HIN200 protein family, was originally identified as a tumor suppressor. ${ }^{5}$ The AIM2 gene has a microsatellite instability site that results in its inactivation in many tumors. ${ }^{6}$ Since its function as cytosolic DNA sensor was disclosed, research on AIM2 has predominantly focused on its role in innate immunity when assembled as an inflammasome. ${ }^{7}$ The AIM2 inflammasome is important in sensing both bacterial and viral pathogens and triggering anti-microbial innate immunity. ${ }^{8}$ Evidence has shown that the AIM2 inflammasome can be activated by Fransicella tularensis, ${ }^{9-11}$ Listeria monocytogenes, ${ }^{11}$ Aspergillus fumigatus, ${ }^{12}$ Streptococcus pneumoniae, ${ }^{13}$ Porphyromonas gingivalis, ${ }^{14}$ vaccinia virus, ${ }^{11}$ human papillomavirus, ${ }^{15}$ and mouse cytomegalovirus. ${ }^{11}$ With regard to Salmonella, although previous work has reported that AIM2-deficient murine bone marrow-derived macrophages respond with interleukin

\footnotetext{
${ }^{1}$ Key Laboratory of Zoonosis, Ministry of Education, College of Veterinary Medicine \& The First Hospital, Jilin University, Changchun, China. Correspondence: Y-J Yang (youngjune@jlu.edu.cn)

${ }^{2}$ These authors contributed equally to this work.

Received 17 June 2015; accepted 16 November 2015; published online 3 February 2016. doi:10.1038/mi.2015.142
} 

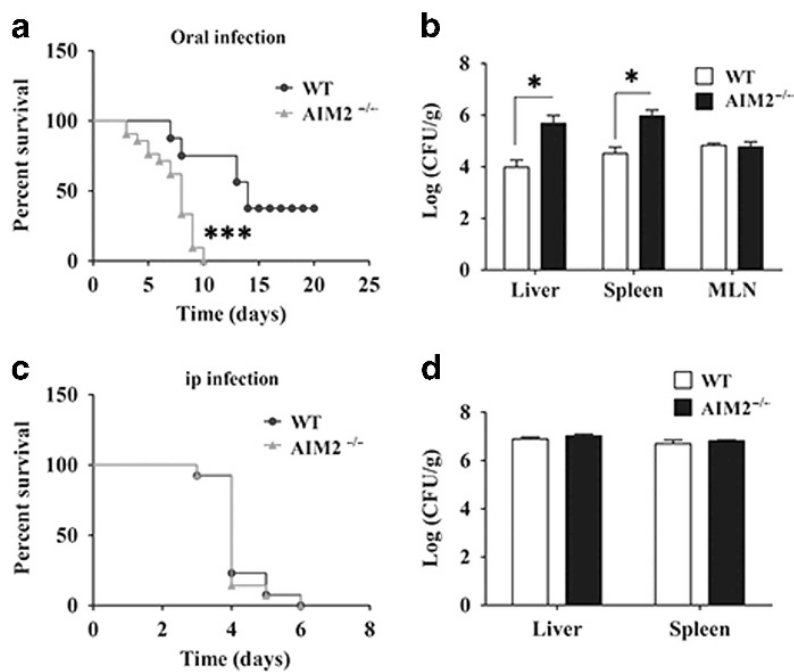

Figure 1 Absent in melanoma-2 (AIM2) deficiency increases the susceptibility to oral but not to systemic Salmonella infection. Wild-type (WT) and AIM2 ${ }^{-1-}$ mice were treated with streptomycin before oral administration of $1 \times 10^{8}$ colony-forming units (CFU) of Salmonella $(n=15$ each group), (a) survival and (b) bacterial dissemination in WT and AIM2 ${ }^{-1-}$ mice was determined at day 5 post infection ( $n=5$ each group). WT and AIM2 $2^{-1-}$ mice intraperitoneally infected with $5 \times 10^{4}$ CFU Salmonella ( $n=13$ each group), (c) survival and (d) bacterial dissemination in WT and AIM2 ${ }^{-1-}$ mice was determined at day 3 post infection ( $n=5$ each group). $P<0.05,{ }^{* \star *} P<0.001$.

(IL) $-1 \beta$ secretion that is similar to that of wild-type (WT) macrophages, ${ }^{11}$ the biological implications of AIM2 during Salmonella infection have not been characterized in vivo.

In this study, we reveal a role for AIM2 in maintaining intestinal barrier function during Salmonella infection. We found that AIM2 protected mice against Salmonella colitis and acted through Akt activation to promote the expression of tight junction proteins that are essential for maintaining intestinal barrier integrity.

\section{RESULTS}

\section{AIM2 protects mice from mucosal but not systemic Salmonella infection}

To explore the possible involvement of AIM2 in the host response to Salmonella infection, we initially examined the mortality rate of WT and AIM2-deficient (AIM2 ${ }^{-1-}$ ) mice following the oral administration of $10^{8}$ colony-forming units (CFU) of Salmonella strain SL1344. WT and AIM2-deficient mice were treated with streptomycin before infection. Compared with WT mice, AIM2 ${ }^{-/-}$mice displayed significantly higher mortality (Figure 1a). On day 10 post infection (p.i.), all of the AIM2 $2^{-1-}$ mice had died, whereas $69 \%$ of the WT control mice remained alive. To further characterize the role of AIM2 in host defense against Salmonella, the effect of AIM2 on bacterial burden was assessed in WT and AIM2 ${ }^{-/-}$ mice. On day 5 p.i., AIM2 ${ }^{-1-}$ mice showed increased bacterial loads in the spleen and liver (Figure 1b), which indicated that $\mathrm{AIM} 2^{-1^{-}}$mice were more susceptible to bacterial dissemination. After oral ingestion, Salmonella crosses the intestinal barrier, disseminates from the GI tract to MLNs, and colonizes systemic sites. ${ }^{16}$ Therefore, this event might be a consequence of either increased penetration and translocation of the epithelial layer by invasive Salmonella or impaired clearance of infection at systemic sites. Hence, we performed intraperitoneal infection of WT and $\mathrm{AIM} 2^{-/-}$mice to determine whether there is an impaired clearance of infection in $\mathrm{AIM} 2^{-1-}$ mice. Interestingly, we observed identical lethality in WT and $\mathrm{AIM} 2^{-1-}$ mice (Figure 1c) and similar bacterial loads in spleen and liver (Figure 1d), which indicated that WT and AIM2 $2^{-1-}$ mice have a similar capacity to eliminate Salmonella systemic infection. Together, these data suggested that AIM2 might have an important role in the GI tract and contribute to the limitation of lethal colitis and bacterial dissemination during Salmonella mucosal infection.

\section{AIM2 is critical in limiting Salmonella colitis}

To determine whether AIM2 deficiency affects Salmonella colitis, the experiment was repeated with the administration of $5 \times 10^{7}$ CFU of Salmonella to study the phenotype of AIM2 ${ }^{-1-}$ mice under milder conditions. We found that AIM2 ${ }^{-1-}$ mice suffered from a greater degree of body weight loss from day 2 onward (Figure 2a). To further assess the severity of colitis, spleen and cecum weights were measured in WT and $\mathrm{AIM} 2^{-l-}$ mice on day 2 p.i. AIM2 ${ }^{-1-}$ mice exhibited severe splenomegaly (Figure $2 \mathbf{b}$ ) and cecal weight loss (Figure 2c). Simultaneously, these clinical assessments were validated by the histological examination of the cecum using hematoxylin and eosin ( $\mathrm{H} \& \mathrm{E})$ staining (Figure 2d). Histological evaluation of the extent of epithelial damage, goblet cell deletion, submucosal edema, and inflammatory cell infiltration revealed that $\mathrm{AIM} 2^{-/-}$mice displayed significantly severe intestinal damage and exacerbated intestinal inflammation. Subsequently, we examined the bacterial load at the mucosal site and systemic site. The AIM2 ${ }^{-1-}$ mice had a higher bacterial load in the cecum, MLN, spleen, and liver, indicating a higher bacterial dissemination in AIM2 ${ }^{-1-}$ mice (Figure 2e).

To gain additional evidence of the role for AIM2 in limiting intestinal colitis, we measured the pro-inflammatory mediator production in the ceca of Salmonella-infected WT and AIM2 ${ }^{-1-}$ mice on day 2 p.i. The amounts of pro-inflammatory cytokines/ chemokines, including TNF $\alpha$, IL- 6 , IL- $1 \beta$, IFN- $\gamma, \mathrm{KC}, \mathrm{CCL} 5$, and CXCL10, in the cecum were significantly higher in AIM2 $2^{-1-}$ mice compared with WT mice (Figure 3a-g). However, $\mathrm{AIM}^{-1-}$ mice and WT mice had comparable levels of CCL2 production in cecum (Figure $3 \mathbf{h}$ ). Additionally, Cox-2 and iNOS, two enzymes associated with inflammation, were also elevated in AIM2 ${ }^{-1-}$ mice (Figure 3i). At a later time point, both $\mathrm{WT}$ and $\mathrm{AIM} 2^{-/-}$mice developed severe disease, and AIM2 ${ }^{-1-}$ mice had a higher histological score of the cecum on days 3, 4, and 5 p.i compared with WT mice (Supplementary Figure $2 \mathrm{~S}$ online). Collectively, the extensive epithelium damage with significant inflammation present in the cecum and higher bacterial dissemination of AIM2 $2^{-1-}$ mice indicated that AIM2 is critical for protection against Salmonella colitis. 

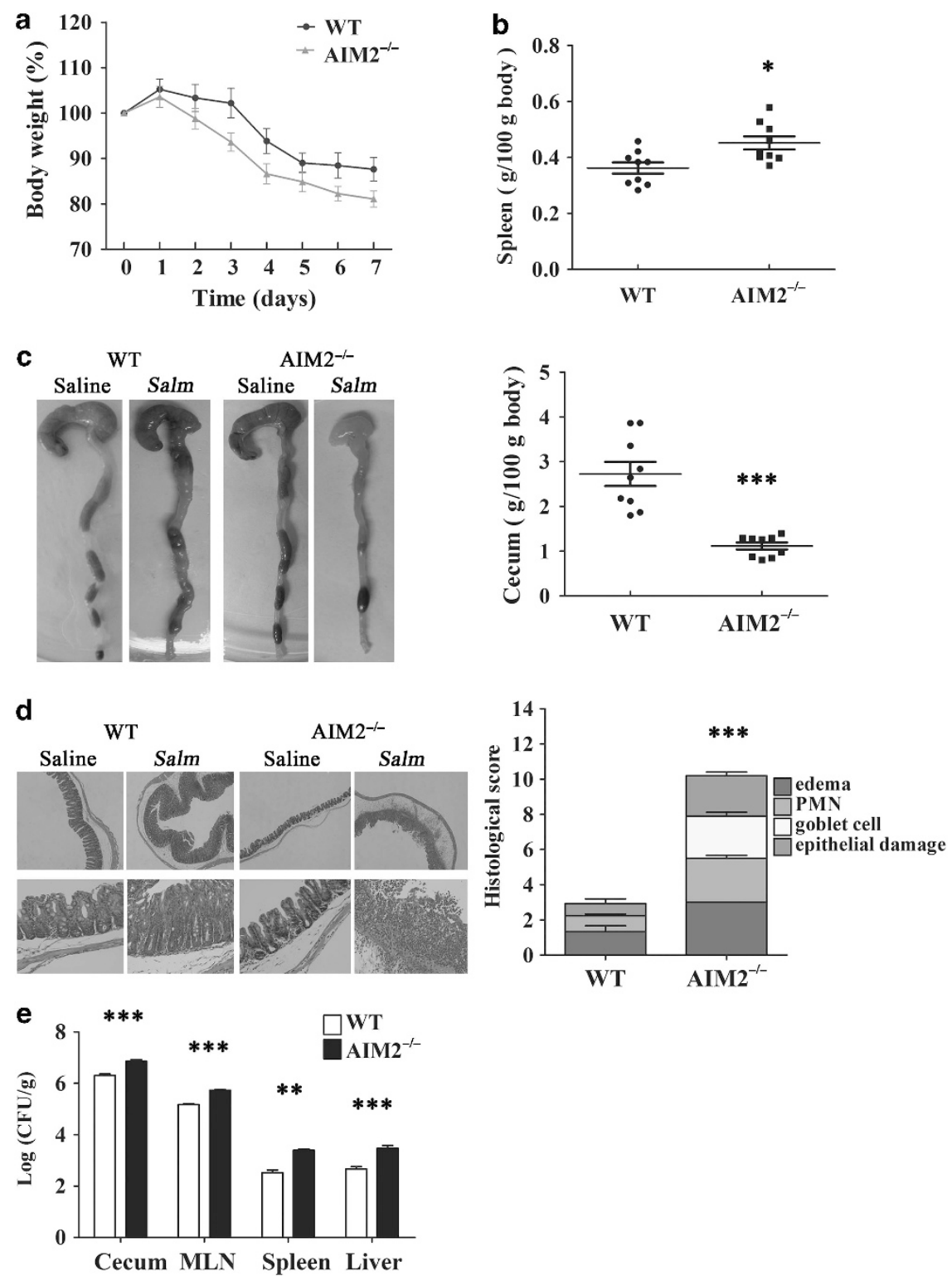

Figure 2 Absent in melanoma-2 (AIM2) deficiency leads to increased intestinal damage during Salmonella mucosal infection. Wild-type (WT) and AIM2 ${ }^{-1-}$ mice were treated with streptomycin before the oral administration of $5 \times 10^{7}$ colony-forming units (CFU) of Salmonella for 2 days $(n=9)$. (a) Body weight loss. (b) Spleen weight. (c) Gross image and weight of cecum. (d) Hematoxylin and eosin (H\&E) staining of the cecum: upper panel, magnification $\times 40$, lower panel, magnification, $\times 100$. (e) Bacterial load in the cecum, mesenteric lymph node $(M L N)$, spleen, and liver. ${ }^{\star} P<0.05$, ${ }^{\star *} P<0.01,{ }^{\star * \star} P<0.001$.

\section{AIM2 is required for maintaining intestinal tissue homeostasis}

Maintaining tissue homeostasis is vital during infection and inflammation. Tissue homeostasis is the regulation of cell proliferation and cell death, also known as cell turnover. Loss of intestinal epithelial cell (IEC) homeostasis negatively affects intestinal barrier function. ${ }^{17}$ To determine whether there is a difference in cell proliferation and apoptosis that can explain the higher susceptibility of AIM2 $2^{-1}$ mice to Salmonella colitis, we first examined cell proliferation by immunofluoresence staining for PCNA, a nuclear antigen for proliferating cells. Our results revealed that the epithelial crypts presented with more PCNA-positive cells in naive $\mathrm{AIM} 2^{-/-}$mice compared with WT mice; however, the level of epithelial cell proliferation significantly decreased following oral administration of Salmonella in AIM2 $2^{-1-}$ mice (Figure 4a). Subsequently, TUNEL (terminal deoxynucleotidyl transferase-mediated dUTP nick-end labeling) staining of histological sections of cecum showed that AIM2 $2^{-1-}$ mice had greatly increased numbers of apoptotic cells in the epithelium and lamina propria (LP) on day 2 p.i, whereas WT mice showed only a mild increase in apoptotic cells in the epithelium (Figure 4b). Apoptosis was further confirmed by immunoblotting for cleaved caspase-3 in cecal lysates, 

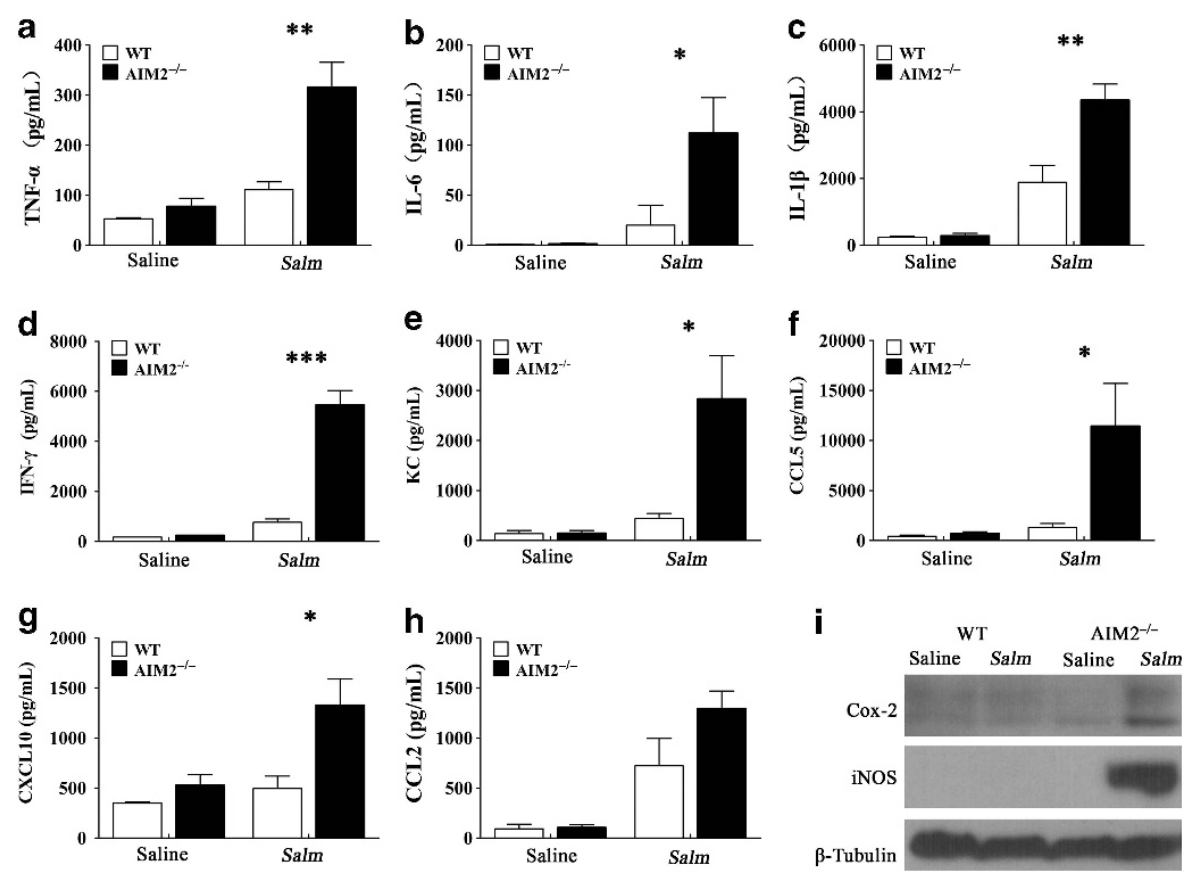

Figure 3 Absent in melanoma-2 (AIM2) deficiency results in elevated pro-inflammatory mediator production during Salmonella mucosal infection. On day 2 post infection, pro-inflammatory mediators in cecum were examined ( $n=9$ each group). (a) TNF $\alpha$; (b) IL-6; (c) IL-1 $\beta$; (d) IFN- $\gamma ;$ (e) KC; (f) CCL5; (g) CXCL10; (h) CCL2 and (i) levels of iNOS and Cox-2 expression in the cecum were examined by western blotting. ${ }^{\star} P<0.05,{ }^{* *} P<0.01,{ }^{* \star \star} P<0.001$.

whereas AIM2 $2^{-1-}$ mice had a higher level of cleaved caspase-3 production (Figure 4c). Collectively, these results revealed that AIM $2^{-1-}$ mice have a defect in maintaining enterocyte proliferative/apoptotic homeostasis.

\section{AIM2 restricts the early paracellular invasion of Salmonella}

The significantly decreased proliferation and increased apoptosis in AIM2 $2^{-1-}$ mice on day 2 p.i maybe the result of exacerbated inflammation; however, the deregulation of basal proliferation in $\mathrm{AIM} 2^{-1-}$ mice may contribute to bacterial dissemination, consequently exacerbating damage and inflammation in the GI tract. To explore this possibility, $\mathrm{WT}$ and $\mathrm{AIM} 2^{-1-}$ mice were treated with streptomycin before the oral administration of $5 \times 10^{7} \mathrm{CFU}$ of Salmonella. Bacterial burden was assessed in the cecal LP and lymph nodes at $3 \mathrm{~h}$ p.i. There were significantly more bacteria detected in both sites of AIM2 $2^{-1-}$ mice compared with the counterparts of WT mice (Figure 5a). This result implied that the absence of AIM2 leads to a defect in the epithelial barrier and confers susceptibility to early bacterial invasion. Pathogens can engage different mechanisms for breaching the epithelial barrier. We hypothesized that the initial increase in bacterial invasion from the GI lumen into the LP in AIM2 $2^{-1-}$ mice may be attributable to a functional defect in epithelial cells. To investigate this possibility, an AIM2-overexpressing Caco-2 cell monolayer was used to analyze the translocation of Salmonella (Figure $5 \mathbf{b}$ ). As expected, the bacterial translocation was significantly decreased in AIM2-overexpressing Caco-2 cells (Figure 5c) but not in Akt inhibitor pretreated cells.
Bacteria can cross epithelial surfaces through paracellular and/or transcellular mechanisms. We thus assessed the intracellular bacterial loads of epithelial cells. Interestingly, there was no significant difference in bacterial load between AIM2-overexpressing Caco-2 cells and vector-transfected control cells (Figure 5d). Therefore, AIM2 might restrict bacterial paracellular penetration through the epithelial lining during early Salmonella infection.

\section{AIM2 promotes tight junction protein expression and decreases epithelial permeability}

The integrity of the GI epithelium is sustained through cell-cell junctions. The tight junction is the major type of cell-cell junction and is composed of scaffolding proteins, including Claudin, Occludin, and Zonula occludens (ZO)-1. The tight junction acts as a permeable barrier against microbes. Based on the data above, we set out to characterize the effects of AIM2 on tight junction and the paracellular permeability of the epithelial lining. Compared with WT mice, the basal levels of Claudin3 (Figure 6a-c) and Occludin (Figure 6d) in $\mathrm{AIM}^{-1-}$ mice were lower whereas there was no difference in the basal level of ZO-1 (Figure 6e). Following the oral administration of Salmonella, there was a decrease in Claudin 3 and Occludin in WT mice, although there was no additional decrease in Claudin3 or Occludin in $\mathrm{AIM} 2^{-1-}$ mice which might due to the already lower level of tight junction protein expression. Moreover, before or after Salmonella challenge, AIM2-overexpressing Caco-2 cells had a higher level of Claudin3 expression and a lower epithelial permeability 

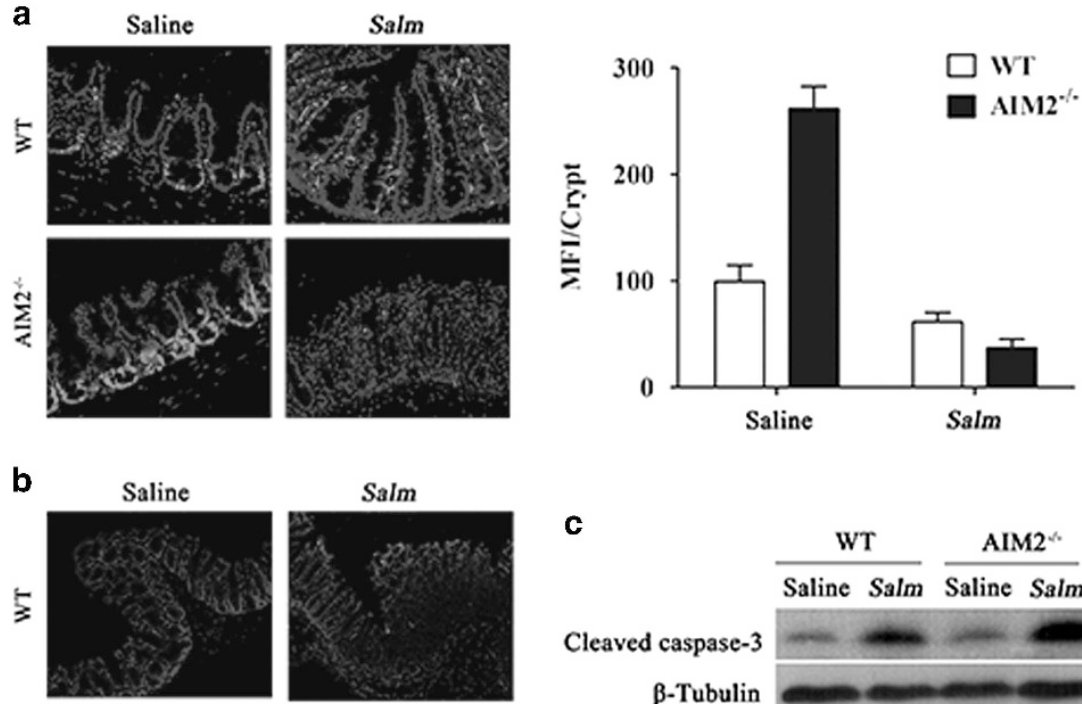

C
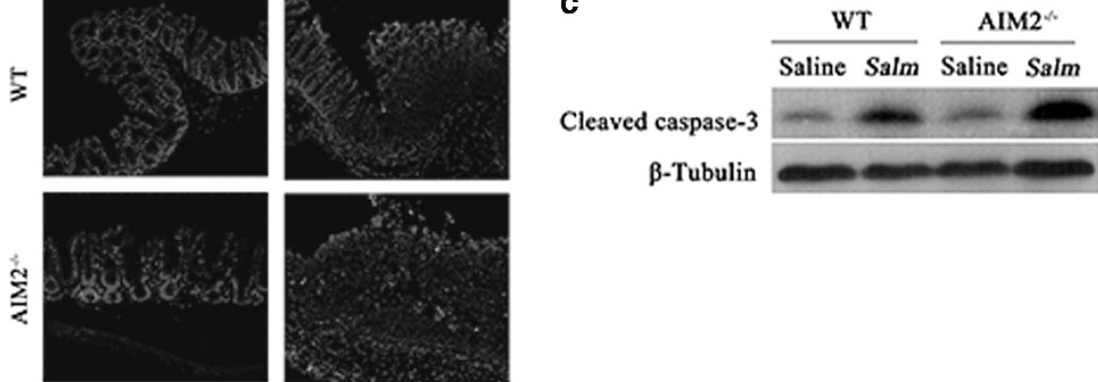

Figure 4 Absent in melanoma-2 (AIM2) deficiency leads to perturbed epithelial cell proliferation and apoptosis following Salmonella oral challenge. (a) Immunofluorescence was performed on cecum sections derived from wild-type (WT) and AIM2 ${ }^{-1}$ mice on day 2 post Salmonella oral infection for the examination of proliferating cells (PCNA). (b) Apoptotic cells were identified in the cecum by terminal deoxynucleotidyl transferase-mediated dUTP nickend labeling (TUNEL) staining. (c) Levels of cleaved caspase-3 expression in cecum lysates were determined by immunoblotting. Magnification, $\times 200$.

a
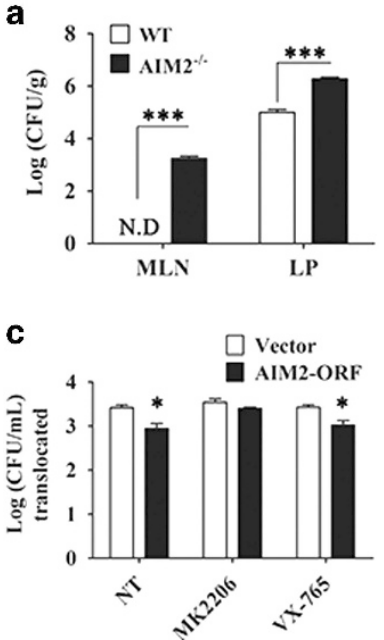

b
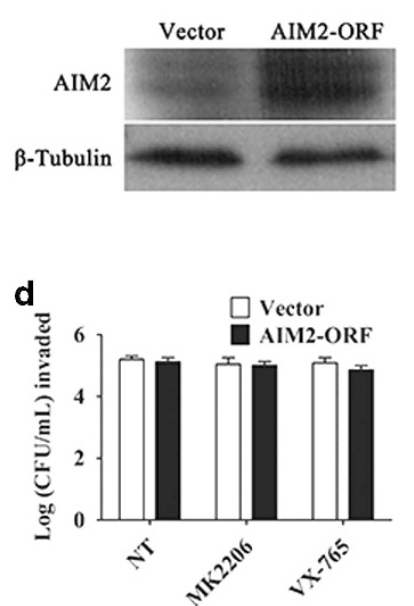

Figure 5 Absent in melanoma-2 (AIM2) restricts Salmonella dissemination and translocation. (a) Bacterial dissemination in the mesenteric lymph node (MLN) and lamina propria (LP) of wild-type (WT) and AIM2 ${ }^{-l-}$ mice following Salmonella infection for $3 \mathrm{~h}(n=8$ each group). (b) AIM2 expression. Cells were treated with Akt and caspase-1 inhibitor, respectively; and bacterial translocation (c) and invasion (d) in vector-transfected Caco-2 cells and AIM2-overexpressing Caco-2 cells were determined $(n=3)$. ND stands for not detected. ${ }^{*} P<0.05$, ${ }^{* * \star} P<0.001$

compared with vector-transfected cells (Figure 6f,g), this effect can be reduced by an Akt inhibitor but not by a caspase-1 inhibitor. Thus, these results indicated that AIM2 promotes the expression of tight junction proteins, and the loss of AIM2 leads to the deregulation of tight junction, consequently making the epithelial barrier fragile enough to be disrupted by Salmonella. Permeability through the paracellular space can be measured by evaluating the trans-epithelial resistance (TER), a hallmark of epithelial integrity. There was a higher TER across monolayer in AIM2-overexpressing Caco-2 cells compared with control cells, which indicated that AIM2 overexpression resulted in deceased permeability and a tightened epithelial barrier (Figure 6h). Collectively, these data suggested that AIM2 protects against Salmonella infection via the enhancement of tight junction barrier function.

\section{Akt mediates AIM2-induced enhancement of tight junction proteins}

Previous reports have indicated that Akt alters the expression of tight junctions. ${ }^{18-20}$ AIM2-overexpressing cells had a higher level of Claudin3 expression (Figure 6f), reduced bacterial translocation (Figure 5c), and increased trans-epithelial electrical resistance (Figure $\mathbf{6 g}$ ). However, this effect can be diminished by an Akt inhibitor but not by a Caspase-1 inhibitor. Thus, we evaluated whether Akt activity is affected by AIM2 and whether Akt mediates the AIM2-induced increase in expression of tight junction proteins. Our results showed that AIM2-overexpressed Caco-2 cells had a higher level of Akt phosphorylation compared with vector-transfected cells (Figure 7a,b). Salmonella challenge promoted Akt phosphorylation and Claudin3 expression at $120 \mathrm{~min}$ 

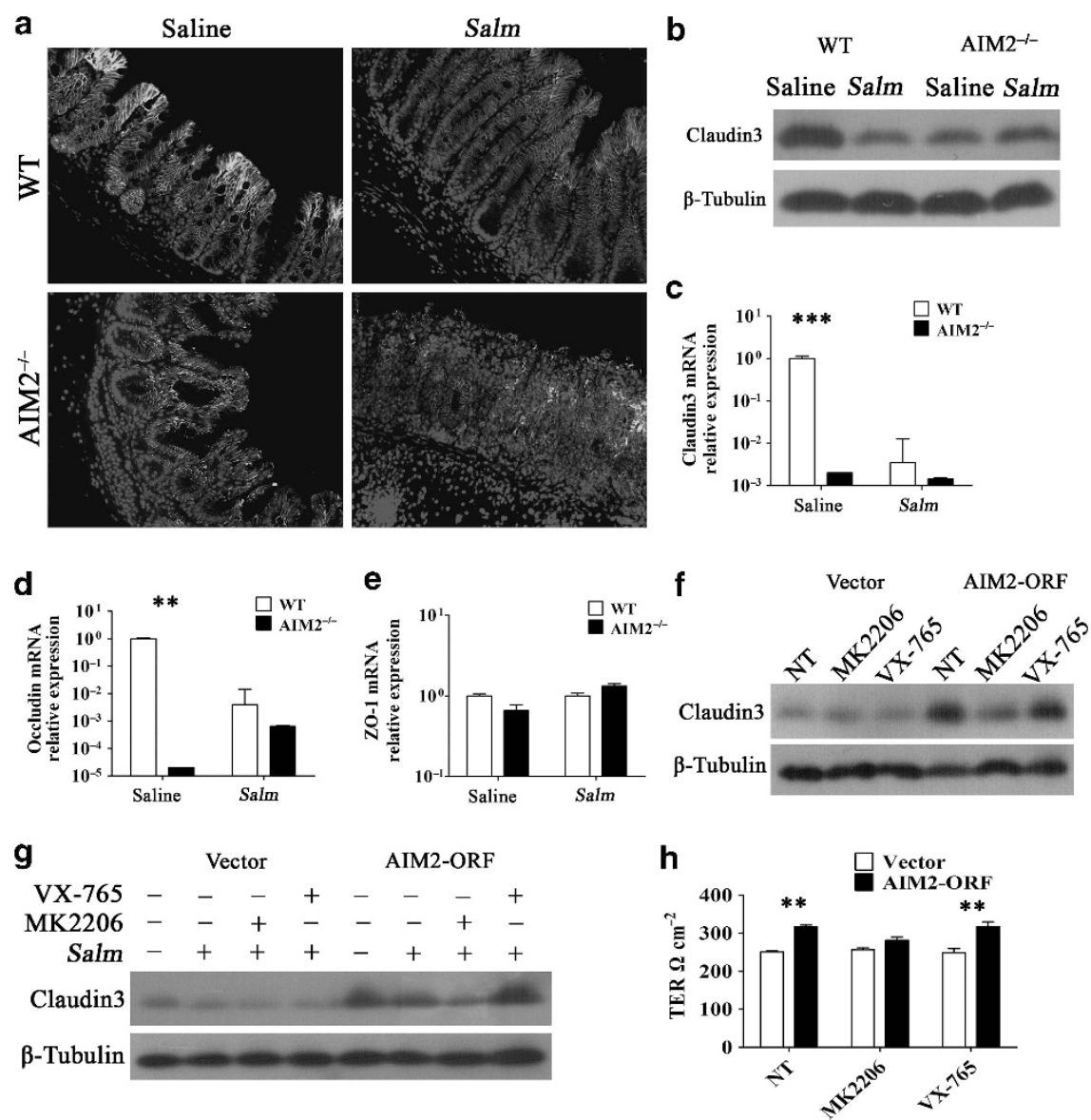

Figure 6 Absent in melanoma-2 (AIM2) enhances the expression of tight junction proteins. Claudin3 expression was examined on cecum sections by (a) immunofluorescence and (b) immunoblotting. The mRNA levels of (c) Claudin3, (d) Occludin, and (e) Zonula occludens (ZO-1) in cecum were examined by real-time PCR. The data were normalized to GAPDH expression and are showed as the fold increase in mRNA. (f) Claudin3 expression levels in Caco-2 cells and AIM2-overexpressing Caco-2 cells were determined. (g) Levels of Claudin3 expression in AIM2-overexpressed Caco-2 cells and vector-transfected cells were determined by western blotting following Salmonella infection for $30 \mathrm{~min}$. (h) Trans-epithelial resistance (TER) levels in Caco-2 cells and AIM2-overexpressing Caco-2 cells were determined. ${ }^{* \star} P<0.01,{ }^{* * *} P<0.001$.

(Figure 7b). Although the basal level of Akt phosphorylation in vector-transfected cells remained very low or undetectable up to $60 \mathrm{~min}$ after Salmonella challenge, inhibition of Akt phosphorylation accompanied by decreased Claudin3 expression was observed in AIM2-overexpressing cells. Therefore, a short exposure of cells to Salmonella inhibited Akt activity and Claudin 3 expression, whereas prolonged Salmonella treatment promoted Akt activity and Claudin3 expression. Moreover, pretreatment with an Akt inhibitor prevented the induction of Akt activation and Claudin3 expression by Salmonella (Figure 7c). Collectively, these results indicated that AIM2-induced enhancement of tight junction proteins may be mediated through Akt.

\section{DISCUSSION}

Acting as a DNA sensor, AIM2 is assembled as an inflammasome and is involved in sensing various bacterial and viral pathogens. However, the biological implications of AIM2 during Salmonella infection remain unknown. We orally infected mice and found that AIM2 ${ }^{-1-}$ mice were more susceptible to Salmonella infection, as indicated by increased mortality and bacterial dissemination. This observation highlights the importance of AIM2 in protection against Salmonella infection.

After oral ingestion, Salmonella initially breaches the intestinal epithelium and then disseminates from the GI tract to colonize systemic sites. Therefore, increased susceptibility of AIM2 ${ }^{-1-}$ mice to Salmonella mucosal infection might be a consequence of either an impaired epithelial barrier or an impaired systemic clearance. To further characterize the role of AIM2 in the host defense against Salmonella, we intraperitoneally infected mice. In this situation, AIM2 deficiency did not affect mortality or bacterial dissemination. The discrepancy between oral and intraperitoneal infection suggests that AIM2 may function in the GI tract to defend against Salmonella infection.

The GI tract provides physical and biological barriers against local and foreign bacteria entry into deeper tissue. It is an extremely complex organ and has a unique immune system. Deregulation of the intestinal immune response appears to have 
a

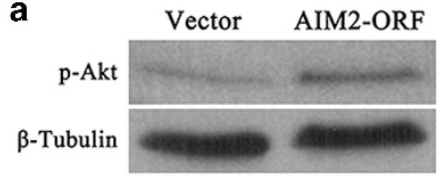

b
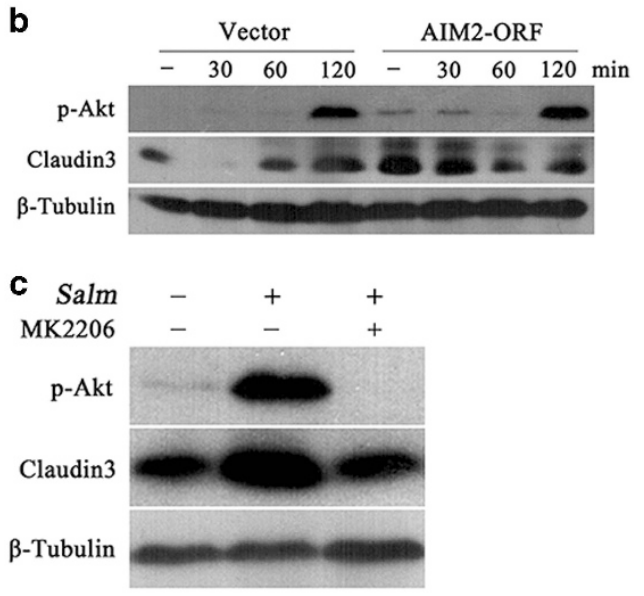

Figure 7 Absent in melanoma-2 (AIM2)-induced enhancement of tight junction proteins is mediated through Akt. (a) Levels of phospho-Akt in both AIM2-overexpressing Caco-2 cells and control cells were measured by western blotting. (b) Levels of phospho-Akt and Claudin3 in both AIM2overexpressed Caco-2 cells and control cells were determined following Salmonella infection for different time points by western blotting. (c) Pretreated Caco-2 cells with Akt inhibitor (MK-2206). Claudin3 expression was examined following Salmonella infection for $120 \mathrm{~min}$ by western blotting.

a principal function in the pathogenesis of inflammatory bowel disease. $^{21} \mathrm{~A}$ healthy gut has evolved diverse regulatory mechanisms that tightly control the immune reaction and cooperate to maintain intestinal immune homeostasis. ${ }^{22,23}$ For example, the Toll-like receptor and Nod-like receptor family members are compartmentally expressed throughout the intestine and recognize microbial components. ${ }^{23,24}$ It has been shown that various members of Toll-like receptors and Nodlike receptors are essential for the maintenance of intestinal homeostasis. ${ }^{24-28}$ To explore whether AIM2 regulates the local immune response in the GI tract, a Salmonella colitis model was established by the oral administration of $5 \times 10^{7} \mathrm{CFU}$ of Salmonella. Our results showed that AIM2 has the potential to be a pattern recognition receptor that limits gut inflammation. AIM2 ${ }^{-1-}$ mice were more susceptible to Salmonella mucosal infection and suffered from heavier intestinal damage with higher pro-inflammatory mediator production. The uncontrolled inflammatory response and the exorbitant intestinal damage in AIM2 ${ }^{-1-}$ mice indicate an essential contribution of AIM2 in limiting inflammatory response and preserving intestinal immune homeostasis. Thus, it is worthwhile to investigate how intestinal immune homeostasis is impaired in the absence of AIM2.

Maintaining intestinal immune homeostasis and generating a proper inflammatory response to a pathogen depend on the integrity of the intestinal barrier. Epithelial cell turnover is required in sustaining intestinal barrier function. ${ }^{29}$ Cell turnover, also known as tissue homeostasis, is the regulation of cell proliferation and cell death and has an important role in maintaining normal tissue function and architecture. ${ }^{30}$ IECs at the villus tips constantly undergo cell death and cell shedding, which contributes to epithelial cell turnover and prevents persistent bacterial colonization. ${ }^{1,31}$ These cells are constantly renewed from cryptic stem cells. If the death and renewal homeostasis of the GI epithelium is broken, then there is an increased risk of microbial infection, tissue injury, or hyperplasia. Our results showed that naive AIM2 ${ }^{-1-}$ mice have increased epithelial cell proliferation and a similar level of epithelial cell apoptosis. Following Salmonella challenge, AIM2 ${ }^{-1-}$ mice were unable to respond normally compared with WT mice, as indicated by the significantly decreased proliferation and increased apoptosis on day 2 p.i. These results suggest that AIM2 is required to maintain the proliferation/ apoptosis homeostasis of IEC.

Although we cannot exclude the possibility that abnormal epithelial proliferation/apoptosis in AIM2 ${ }^{-1-}$ mice on day 2 p.i is a consequence rather than the cause of exacerbated inflammation, the deregulated proliferation in naive AIM $2^{-1-}$ mice may contribute to the higher bacterial dissemination and excessive inflammation response. To explore this possibility, bacterial burden was assessed in the cecal LP and lymph nodes as early as $3 \mathrm{~h}$ p.i. Higher bacterial loads in both sites of AIM2 ${ }^{-1-}$ mice suggest that AIM2 deficiency results in an impaired epithelial barrier. The pathogen can engage IECs or LP phagocytes for transversing the epithelium to access the LP. Based on a previous report that AIM2-deficient macrophages respond to Salmonella with normal IL-1 $\beta$ production ${ }^{11}$ and our data regarding increased IEC proliferation in naive AIM2 ${ }^{-1-}$ mice, we hypothesized that the initially increased bacterial invasion from the GI lumen into the LP in AIM2 ${ }^{-1-}$ mice may be attributable to abnormal IEC function. This hypothesis was tested using a human intestinal epithelial cell line, Caco-2. As expected, AIM2 overexpression restricted Salmonella translocation across epithelial cells. Moreover, we found that the altered capacity of paracellular rather than transcellular penetration was responsible.

The integrity of the gut epithelium is also sustained through tight junction, which acts as a permeable barrier in the defense against foreign antigens and microbes. A loss of tight junction structure and function is frequently observed in inflammatory bowel disease, leaky diarrhea, and epithelium-derived cancers. ${ }^{32-34}$ Disruption of intestinal tight junction by microbes benefits their dissemination inside the host. We thus examined the effects of AIM2 on the expression of tight junction proteins. Our results showed that the basal level of Claudin 3 and Occludin in naive AIM2 ${ }^{-1-}$ mice was significantly lower compared with those in WT mice. This effect was confirmed in vitro. Moreover, AIM2 overexpression resulted in an increased TER. Collectively, these data suggest that AIM2 contributes to maintain the integrity of intestinal barrier through enhancing the expression of tight junction proteins. Due to the disrupted integrity of the intestinal barrier, AIM2 ${ }^{-1-}$ mice are more readily invaded by pathogens and consequently exhibit a higher mortality rate, bacterial 
dissemination, and overwhelming inflammation. Although barrier defects were found in AIM2 ${ }^{-1-}$ mice, spontaneous inflammation was not observed. This result suggests that intestinal barrier dysfunction is insufficient to cause spontaneous intestinal inflammation. There might be a compensatory mechanism helping to maintain homeostasis in uninfected AIM2 $2^{-1-}$ mice under uninfected condition. However, in a hostile environment, AIM2 $2^{-/-}$mice are more readily infected due to the fragile intestinal barrier.

Subsequently, we observed that AIM2-overexpressing Caco-2 cells had a higher level of Akt phosphorylation compared with that in control cells. Salmonella infection activated phospho-Akt at $120 \mathrm{~min}$, and a corresponding increase in Claudin 3 expression was observed. The chemical inhibition of Akt activation prevented the induction of Claudin 3 by Salmonella challenge. Thus, we found that AIM2 regulates Akt signaling, thus mediating the enhancement of tight junction proteins. How AIM2 regulates Akt phosphorylation in epithelium upon Salmonella challenge remains elusive. Recently, a report has shown that AIM2 suppresses colon tumorigenesis by interfering with Akt activation in an inflammasome-independent manner. ${ }^{35}$ In our study, IL- $1 \beta$ production was higher and IL-18 transcript (Supplementary Figure S3) remained comparable in the cecum of AIM2 ${ }^{-1-}$ mice compared with WT mice after Salmonella challenge. However, we still cannot make sure that AIM2 contributes to the maintenance of intestinal integrity in an inflammasomeindependent manner. To address this possibility, caspase-1deficient mice and mice deficient in both caspase-1 and AIM2 need to be analyzed with respect to inflammatory responses and tight junctions.

Salmonella have evolved various mechanisms to penetrate the epithelial monolayer, such as dysregulating tight junctions through effector proteins. It was previously reported that SopB, SopE, SopE2, and SipA can induce ruffling of cell membrane and change the localization and expression of ZO-1 and occludin, facilitating bacterial invasion. ${ }^{36}$ Salmonella also secrete AvrA to stabilize tight junctions. ${ }^{37}$ In this study, we found that Claudin3 expression gradually decreased until $60 \mathrm{~min}$ after Salmonella infection, and then increased at $120 \mathrm{~min}$. This dynamic expression of tight junction protein might be due to the regulation of a panel of effectors, which facilitates pathogen invasion or intracellular survival. AIM2 enhances intestinal barrier function to restrict Salmonella invasion. There also might be some Salmonella effectors, which promote invasion by hampering AIM2 signaling.

In summary, our findings establish a role for AIM2 in maintaining intestinal barrier integrity, pave the way for future studies aiming to better define the role of AIM2 in regulating innate immunity, and provide clues regarding the control of intestinal diseases.

\section{METHODS}

Mice. AIM2 ${ }^{-1-}$ mice were purchased from the Jackson Laboratory (Bar Harbor, ME) and were subsequently backcrossed onto the C57BL/6J background for another eight generations. Heterozygous breeding pairs were used to generate WT mice. WT and AIM2 $2^{-1-}$ mice carry a mutant Nramp1 allele (Supplementary Figure S1). All animal studies were conducted according to the experimental practices and standards approved by the Animal Welfare and Research Ethics Committee at Jilin University.

In vivo infections. Six- to eight-week-old sex-matched mice were used in this study. The Salmonella-induced colitis model was established as previously described. ${ }^{38}$ Briefly, following the administration of $20 \mathrm{mg}$ of streptomycin per mouse, WT and AIM2-deficient mice were orally challenged with $5 \times 10^{7}$ CFU of Salmonella strain SL1344 for $3 \mathrm{~h}, 2$ days, or 5 days p.i. Log CFU per organ values were determined. Cecum inflammation was analyzed 2 days p.i. For systemic infection, mice were intraperitoneally infected with $5 \times 10^{4} \mathrm{CFU}$ of Salmonella and log $\mathrm{CFU}$ per organ values were determined at 3 days p.i. For the survival study, WT and AIM2 $2^{-1-}$ mice were orally infected with $1 \times 10^{8} \mathrm{CFU}$ or intraperitoneally infected with $5 \times 10^{4} \mathrm{CFU}$ of Salmonella. Bacterial loads were measured as follows. Spleen, liver, and MLN were weighed and homogenized in cold phosphate-buffered saline (PBS) at a ratio of $4 \mathrm{~mL}$ per gram of tissue; for the cecum, tissue was washed free of feces and weighed and subsequently homogenized in cold PBS. For the LP, the bacterial load was examined as previously described. ${ }^{39}$ Briefly, the cecum was washed free of feces with PBS three times and then cut into small pieces and incubated in Hank's Balanced Salt Solution containing $5 \mathrm{~mm}$ EDTA, $10 \mathrm{~mm}$ hydroxyethyl piperazine ethanesulfonic acid, and $5 \%$ fetal bovine serum at $37^{\circ} \mathrm{C}$ for $20 \mathrm{~min}$, followed by vigorous vortexing to remove the epithelial cell layers. This process was repeated two times. The remaining tissue was homogenized in sterile PBS, and serial dilutions were plated on lysogeny broth agar.

Histology and immunofluorescence. At 2 days p.i., the cecum was harvested, washed free of feces with cold PBS, it was then fixed in formalin and embedded in paraffin; $5 \mu \mathrm{m}$ sections were used for $\mathrm{H} \& \mathrm{E}$ or immunofluorescence staining. Histological assessment was performed randomly using a scoring system as previously described. ${ }^{38}$ Immunostaining was performed using anti-PCNA (1:50, Santa Cruz Biotechnology, Santa Cruz, CA) and anti-Claudin3 (1:100, Abcam, Cambridge, MA) antibodies. Epithelial cell apoptosis was analyzed by TUNEL staining using a commercial kit (KeyGEN BioTECH, Nanjing, China).

Western blotting analysis. The ceca of mice were harvested and washed free of feces with PBS, and then homogenized in lysis buffer containing 1\% Triton X-100, 50 mм Tris- $\mathrm{HCl}(\mathrm{pH} 7.4), 150 \mathrm{~mm} \mathrm{NaCl}$, $0.1 \mathrm{mM} \mathrm{Na}_{3} \mathrm{VO}_{4}, 0.1 \mathrm{~mm} \mathrm{NaF}, 1 \mathrm{~mm}$ PMSF, $5 \mu \mathrm{g} \mathrm{mL}^{-1}$ Aprotinin, $5 \mu \mathrm{g} \mathrm{mL}^{-1}$ Leupeptin, and $5 \mu \mathrm{g} \mathrm{mL}^{-1}$ Pepstatin A. Homogenates were centrifuged at 13,000 r.p.m. per minute for $30 \mathrm{~min}$ and supernatants were collected and used for immunoblotting analysis. The lysates $(30 \mu \mathrm{g})$ were subjected to $12 \%$ sodium dodecylsulfate polyacrylamide gel electrophoresis and transferred onto polyvinylidene fluoride membranes (Millipore, Billerica, MA). After blocking with 5\% milk, the membrane was blotted with antibodies against iNOS (Abcam), Cox-2 (Abcam), Claudin3 (Abcam), Phospho-Akt (Cell Signaling, Beverly, MA), Cleaved caspase-3 (Cell Signaling), AIM2 (Proteintech, Wuhan, China), and $\beta$-Tubulin (Sungene Biotech, Tianjin, China).

Cytokine analysis. The ceca of mice were harvested, washed free of feces with PBS, and subsequently, weighed and homogenized in cold PBS at a ratio of $4 \mathrm{~mL}$ per gram tissue. The homogenates were centrifuged at 13,000 r.p.m. per minute for $30 \mathrm{~min}$, and supernatants were used for cytokine examination. Cytokines and chemokines in tissue homogenate were detected by an enzyme-linked immunosorbent assay following the R\&D Systems (Minneapolis, MN) instruction.

RNA isolation, reverse transcription-PCR, and real-time PCR. Total RNA was extracted from tissue samples using TRI-Reagent (Sigma-Aldrich, Shanghai, China) according to the manufacturer's instructions. The reverse transcription-PCR was performed using a RT-PCR Kit (TaKaRa, Kyoto, Japan). Quantitative real-time PCR was 
performed on an IQ5 Real-Time PCR Detection System (Bio-Rad Laboratories, Hercules, CA) using SYBR Green PCR Master Mix (Roche Diagnostics, South San Francisco, CA). The levels of cDNA were determined using a standard curve and normalized to GAPDH. Each example was run in triplicate. The relative changes in gene expression were analyzed by the $2^{- \text {Eac }}$ method, and a melting-curve analysis was performed to ensure the specificity of the products. The following primers were used: Claudin $3,5^{\prime}$-CCTAGGAACTGTCC AAGCCG- $3^{\prime}$ and 5' ${ }^{\prime}$-CCCGTTTCATGG TTTGCCTG-3' - Occludin, $5^{\prime}$-CAGCCTTCTGCTTCATCG- ${ }^{\prime}$ and $5^{\prime}$-GTCGGGTTCACTCCC AT TA-3' ZO-1, 5'-GACCTTGAGCAGCCGTCATA-3' and 5' -CCGTAGG CGATGGTCATAGTT- $3^{\prime}$ GAPDH, $5^{\prime}$-CACCCCAGC AAGGACACT GAGCAAG-3' and $5^{\prime}$-GGGGGTCTGGGATGGAAATTGTGAG-3' .

In vitro infection and translocation assay. Caco-2 cells and AIM2overexpressing Caco- 2 cells were seeded at a high density of $5 \times 10^{5}$ cell per transwell filter $(3 \mu \mathrm{m}$ pore size, Thermo Fisher, Odense, Denmark, DM) for bacterial translocation and invasion studies. The medium was refreshed daily until the transepithelial electrical resistance was $\geqslant 250 \Omega \mathrm{cm}^{-2}$, as measured using a Millicell Electrical Resistance System (Millipore). Before infection, the medium was changed to Dulbecco's Modified Eagle's Medium (DMEM)/F12. Salmonella was added at $1 \times 10^{7} \mathrm{CFU}$ per well. For translocation, $1 \mathrm{~h}$ p.i, samples were taken from the basolateral chamber and rates of translocation were determined. To determine infection, $1 \mathrm{~h}$ p.i, the medium was replaced with fresh medium containing $100 \mu \mathrm{g} \mathrm{mL}^{-1}$ gentamycin for $30 \mathrm{~min}$ to kill the extracellular bacteria. The filter was washed apically and basolaterally three times with PBS and then lysed in $1 \mathrm{~mL}$ of $0.1 \%$ Triton X-100 for $10 \mathrm{~min}$. Serial dilutions were plated on lysogeny broth agar plates.

AIM2-overexpressing Caco-2 cell construction. AIM2 overexpression was performed with Lipofection 2000 (Invitrogen, Carlsbad, $\mathrm{CA}$ ) in accordance with the manufacturer's instructions. Caco-2 cells were stably transfected with plasmid pcDNA3.0 or pcDNA3.0 encoding human AIM2. Forty-eight hours post transfection, G418 was added at a final concentration of $500 \mathrm{ng} \mathrm{mL}^{-1}$; the culture medium was replenished every other day until single clones were formed.

Inhibition of Akt and caspase-1 activity in vitro. Cells were pretreated with $1 \mu \mathrm{M}$ Akt inhibitor (MK2206) for $2 \mathrm{~h}$ and $5 \mu \mathrm{M}$ caspase- 1 inhibitor (VX-765) for $45 \mathrm{~min}$.

Statistical analyses. All experiments were independently performed three times in triplicate. The results are showed as mean \pm s.e. The differences between mean values were assessed with ANOVA Dunnett's multiple comparison test and Student's $t$-test, and a value of $P<0.05$ was considered to be statistically significant. Statistics were calculated with the Prism software (GraphPad, La Jolla, CA).

SUPPLEMENTARY MATERIAL is linked to the online version of the paper at http://www.nature.com/mi

\section{ACKNOWLEDGMENTS}

This work was supported by National Natural Science Foundation of China (No. 31372410), National Key Basic Research Program of China (No. 2011CB944404), Changjiang Scholars and Innovative Research Team in University (No. IRT1248).

\section{DISCLOSURE}

The authors declared no conflict of interest.

(c) 2016 Society for Mucosal Immunology

\section{REFERENCES}

1. Kim, M., Ashida, H., Ogawa, M., Yoshikawa, Y., Mimuro, H. \& Sasakawa, C. Bacterial interactions with the host epithelium. Cell Host Microbe 8, 2035 (2010).
2. Corr, S.C. et al. MyD88 adaptor-like (Mal) functions in the epithelial barrier and contributes to intestinal integrity via protein kinase C. Mucosal Immunol. 7, 57-67 (2014).

3. Kaiser, P., Diard, M., Stecher, B. \& Hardt, W.D. The streptomycin mouse model for Salmonella diarrhea: functional analysis of the microbiota, the pathogen's virulence factors, and the host's mucosal immune response. Immunol. Rev. 245, 56-83 (2012).

4. Muller, A.J. et al. Salmonella gut invasion involves TTSS-2-dependent epithelial traversal, basolateral exit, and uptake by epithelium-sampling lamina propria phagocytes. Cell Host Microbe 11, 19-32 (2012).

5. DeYoung, K.L. et al. Cloning a novel member of the human interferoninducible gene family associated with control of tumorigenicity in a model of human melanoma. Oncogene 15, 453-457 (1997).

6. Woerner, S.M. et al. The putative tumor suppressor AIM2 is frequently affected by different genetic alterations in microsatellite unstable colon cancers. Genes Chromosomes Cancer 46, 1080-1089 (2007).

7. Hornung, V. et al. AIM2 recognizes cytosolic dsDNA and forms a caspase1-activating inflammasome with ASC. Nature 458, 514-518 (2009).

8. Franchi, L. \& Nunez, G. AIM2 joins the gang of microbial sensors. Cell Host Microbe 7, 340-341 (2010).

9. Meunier, E. et al. Guanylate-binding proteins promote activation of the AIM2 inflammasome during infection with Francisella novicida. Nat. Immunol. 16, 476-484 (2015).

10. Man, S.M. et al. The transcription factor IRF1 and guanylate-binding proteins target activation of the AIM2 inflammasome by Francisella infection. Nat. Immunol. 16, 467-475 (2015).

11. Rathinam, V.A. et al. The AIM2 inflammasome is essential for host defense against cytosolic bacteria and DNA viruses. Nat. Immunol. 11, 395-402 (2010).

12. Karki, R. et al. Concerted activation of the AIM2 and NLRP3 inflammasomes orchestrates host protection against Aspergillus infection. Cell Host Microbe 17, 357-368 (2015).

13. Fang, R. etal. Type l interferon signaling regulates activation of the absent in melanoma 2 inflammasome during Streptococcus pneumoniae infection. Infect. Immun. 82, 2310-2317 (2014).

14. Park, E., Na, H.S., Song, Y.R., Shin, S.Y., Kim, Y.M. \& Chung, J. Activation of NLRP3 and AIM2 inflammasomes by Porphyromonas gingivalis infection. Infect. Immun. 82, 112-123 (2014).

15. Reinholz, M. et al. HPV16 activates the AIM2 inflammasome in keratinocytes. Arch. Dermatol. Res. 305, 723-732 (2013).

16. Broz, P., Ohlson, M.B. \& Monack, D.M. Innate immune response to Salmonella typhimurium, a model enteric pathogen. Gut Microbes 3, 62-70 (2012).

17. Waldschmitt, N. et al. C/EBP homologous protein inhibits tissue repair in response to gut injury and is inversely regulated with chronic inflammation. Mucosal Immunol. 7, 1452-1466 (2014).

18. de Araujo, W.M., Vidal, F.C., de Souza, W.F., de Freitas, J.C. Jr., de Souza, W. \& Morgado-Diaz, J.A. PI3K/Akt and GSK-3beta prevents in a differential fashion the malignant phenotype of colorectal cancer cells. J. Cancer Res. Clin. Oncol. 136, 1773-1782 (2010).

19. Failor, K.L., Desyatnikov, Y., Finger, L.A. \& Firestone, G.L. Glucocorticoidinduced degradation of glycogen synthase kinase-3 protein is triggered by serum- and glucocorticoid-induced protein kinase and Akt signaling and controls beta-catenin dynamics and tight junction formation in mammary epithelial tumor cells. Mol. Endocrinol. 21, 2403-2415 (2007).

20. Lin, N., Xu, L.F. \& Sun, M. The protective effect of trefoil factor 3 on the intestinal tight junction barrier is mediated by toll-like receptor 2 via a PI3K Akt dependent mechanism. Biochem. Biophys. Res. Commun. 440, 143-149 (2013).

21. Nenci, A. et al. Epithelial NEMO links innate immunity to chronic intestinal inflammation. Nature 446, 557-561 (2007).

22. Maloy, K.J. \& Powrie, F. Intestinal homeostasis and its breakdown in inflammatory bowel disease. Nature 474, 298-306 (2011).

23. Rescigno, $M$. The intestinal epithelial barrier in the control of homeostasis and immunity. Trends Immunol. 32, 256-264 (2011).

24. Magalhaes, J.G., Tattoli, I. \& Girardin, S.E. The intestinal epithelial barrier: how to distinguish between the microbial flora and pathogens. Semin. Immunol. 19, 106-115 (2007).

25. Mathur, R. et al. A mouse model of Salmonella typhi infection. Cell 151, 590-602 (2012). 
26. Natividad, J.M. et al. Commensal and probiotic bacteria influence intestinal barrier function and susceptibility to colitis in Nod1-/-; Nod2-/- mice. Inflamm. Bowel Dis. 18, 1434-1446 (2012).

27. Brandl, K. et al. MyD88 signaling in nonhematopoietic cells protects mice against induced colitis by regulating specific EGF receptor ligands. Proc. Natl. Acad. Sci. USA 107, 19967-19972 (2010).

28. Brun, P. et al. Toll-like receptor 2 regulates intestinal inflammation by controlling integrity of the enteric nervous system. Gastroenterology 145 , 1323-1333 (2013).

29. Peterson, L.W. \& Artis, D. Intestinal epithelial cells: regulators of barrier function and immune homeostasis. Nat. Rev. Immunol. 14, 141-153 (2014).

30. Alexander, B.E. et al. Cell turnover and detritus production in marine sponges from tropical and temperate benthic ecosystems. PLoS One 9 , e109486 (2014).

31. Vereecke, L., Beyaert, R. \& van Loo, G. Enterocyte death and intestinal barrier maintenance in homeostasis and disease. Trends Mol. Med. 17, 584-593 (2011).

32. Zeissig, S. et al. Changes in expression and distribution of claudin 2, 5 and 8 lead to discontinuous tight junctions and barrier dysfunction in active Crohn's disease. Gut 56, 61-72 (2007).
33. Das, P. et al. Comparative tight junction protein expressions in colonic Crohn's disease, ulcerative colitis, and tuberculosis: a new perspective. Virchows Arch. 460, 261-270 (2012).

34. Mees, S.T. et al. Expression of tight and adherens junction proteins in ulcerative colitis associated colorectal carcinoma: upregulation of claudin1, claudin-3, claudin-4, and beta-catenin. Int. J. Colorectal Dis. 24, 361-368 (2009).

35. Wilson, J.E. et al. Inflammasome-independent role of AIM2 in suppressing colon tumorigenesis via DNA-PK and Akt. Nat. Med. 21, 906-913 (2015).

36. Boyle, E.C., Brown, N.F. \& Finlay, B.B. Salmonella enterica serovar Typhimurium effectors SopB, SopE, SopE2 and SipA disrupt tight junction structure and function. Cell Microbiol. 8, 1946-1957 (2006).

37. Liao, A.P. etal. Salmonellatype Ill effector AvrA stabilizes cell tight junctions to inhibit inflammation in intestinal epithelial cells. PLoS One 3, e2369 (2008).

38. Stecher, B., Macpherson, A.J., Hapfelmeier, S., Kremer, M., Stallmach, T. \& Hardt, W.D. Comparison of Salmonella enterica serovar Typhimurium colitis in germfree mice and mice pretreated with streptomycin. Infect. Immun. 73, 3228-3241 (2005).

39. Khounlotham, M. et al. Compromised intestinal epithelial barrier induces adaptive immune compensation that protects from colitis. Immunity 37 , 563-573 (2012). 